\title{
Survey of evidence-based practice among critical care nurses in Denmark
}

Ingrid Egerod, RN, MSN, PhD, Associate Professor, University Hospitals Centre of Nursing and Care Research (UCSF), Rigshospitalet afsnit 7331, DK-2100 Copenhagen O, Denmark.

E-mail: ingrid@ucsf.dk

Key words: critical care evidence-based practice intensive care unit knowledge national survey nursing research utilisation

\section{SUMMARY}

* Evidence-based practice (EBP) has been introduced into hospital settings throughout the world with the goal of information management, cost constraint and optimisation of patient care.

* Although EBP has been defined as the integration of best research evidence with clinical expertise and patient values, many use the term synonymously with research utilisation.

* The aim of the study was to describe the extent of the use of EBP in critical care nursing in Danish hospitals in 2004, and to explore nurses' self-reported attitudes and knowledge of EBP by describing the types of information used by the respondents in clinical decision-making.

* The survey was sent to nurses in each intensive care unit in Denmark. The response rate was $79 \%$ after two reminders.

* The study suggests that critical care nurses use personal experience as their primary source of knowledge, while research-based journals are the least commonly used source. Nonetheless, the nurses displayed a positive attitude toward EBP and a perception that it promotes the profession of nursing.

\section{INTRODUCTION}

In an effort to facilitate the application of research findings in clinical practice, evidence-based practice (EBP) has been introduced into hospitals throughout the world. Although the term EBP has been applied universally, the concept lacks final definition (French 2002; Wang 2004). The term has emerged from evidence-based medicine (EBM), which has been defined as the integration of best research evidence with clinical expertise and patient values (Sackett et al. 2000). In this regard, best research evidence includes patient-centred clinically relevant research, and it is assumed that new evidence may invalidate previously accepted knowledge and replace it with newer and better diagnostic tests and treatments. According to this definition, clinical expertise includes eligibility derived from experience, for example the ability to determine each patient's health state, and personal values and expectations. Patient values are unique preferences, concerns and expectations, which are integrated into clinical decisions to serve the patient. A broader definition of EBP is the integration of scientific facts with value judgments as well as economical considerations (Saarni \& Gylling 2004).

A discourse exists currently as to whether EBP represents a new paradigm, or whether it is a new word for known phenomena, such as research utilisation or quality improvement (DiCenso et al. 2000; French 2002). What appears to be new is that EBP consists of five well-defined steps:

* The formulation of an answerable question;

* Systematic literature search and appraisal of validity;

* Integration of evidence with clinical experience and patient preferences;

* Implementation of the EBP decision

* Evaluation of the patient outcome (DiCenso et al. 2000; Guyatt \& Rennie 2002).

It may be argued that the methodologies for EBP, research utilisation and quality improvement are similar in essence.

EBP relies on the use of research-based literature as the primary source of information. A study looking at the type of information doctors use for clinical decision making shows that textbooks, journals and other existing information is inadequate in clinical practice, and that doctors are most likely to seek information from other doctors (Smith 1996). Similarly, a survey of nurses in western Canada in 1996 showed that nurses use a broad range of practice knowledge, which is experientially-based rather than researchbased (Estabrooks 1998).

It was hypothesised in the present study that Danish critical care nurses rely on personal experience rather than research-based literature for clinical decision making. The aim of the study was to describe the extent of the use of EBP in critical care nursing at Danish hospitals in 2004, in addition to exploring nurses' self-reported attitudes and knowledge of EBP, and determining the types of information used by the respondents when making clinical decisions. The survey was a pilot study for a larger study regarding EBP among cardiac nurses. 


\section{METHOD}

Prior to the study, a network of critical care nurses was established with one or more contact nurses at each Danish intensive care unit (ICU). The purpose of the network was research participation and information sharing. Although critical care certification is not yet mandatory for work in intensive care units, the contact nurses were required to be certified to ensure clinical experience. A second requirement was that the nurses could be reached via e-mail. At the time of the study, 47 nurses volunteered to be contact nurses in the network, and 38 out of 49 Danish intensive care units were represented.

The respondents in this study were selected as a convenience sample. In January 2004, e-mail surveys were sent to each contact nurse in the network $(n=47)$, and postal self-administered questionnaires were sent to one nurse at each of the non-participating intensive care units $(n=11)$. The response rate for nurses in the network was $87 \%$, with $46 \%$ for nurses at non-participating intensive care units, yielding a final response rate of $79 \%$ after two reminders. The desired response rate was greater than $80 \%$ to avoid selection bias (De Vaus 2002). The response rate was high compared with a similar Canadian study in 1996, which had a response rate of $40 \%$ after three reminders (Estabrooks 1998).

Prior to the survey, the researchers knew that some hospitals preferred to use the term research-based practice rather than EBP. In order to encourage participation in the survey, it was decided that EBP should be used in its broadest meaning, to include EBP, evidence-based nursing and research-based practice.

The questionnaire included demographic questions, and questions related to hospital policy, the understanding of EBP, and the source of information used in clinical decision-making.

Among the ethical considerations were anonymity and confidentiality. The survey was not anonymous, as the researchers needed to identify the respondents to post reminders, but the data were handled confidentially and care was taken to protect the respondents' identity (De Vaus 2002).

\section{RESULTS}

\section{Demographics}

The respondents $(n=46)$ were one male and 45 female nurses. $5 \%$ were head nurses, $24 \%$ bedside nurses, while $71 \%$ were nurses with special functions, for example clinical nurse specialist (CNS). The mean age of the population was 45 years and the mean length of experience was 21 years. Danish nurses had been educated as hospital diploma nurses until 2000 when a baccalaureate degree was introduced. Only one nurse in the survey held a baccalaure- ate degree, while $96 \%$ of the sample reported having critical care certification. Half of the sites in the study were university hospitals $(n=23)$, while the other half were rural or non-university hospitals $(n=23)$.

\section{Evidence-based practice as policy}

Table 1 shows the responses related to EBP as policy. The data show that EBP was reported to be formal policy at less than half of the hospitals ( $41 \%$ ) and units (42\%) in the survey. The respondents were more confident about the policy at unit level than at hospital level, as $32 \%$ did not know the hospital policy. Only $16 \%$ of the hospitals offered an introductory course in EBP, and this was mandatory at $4 \%$ of the sites. $65 \%$ of the sites used evidence-based guidelines and half of the sites had a designated nurse (for example clinical nurse specialist) at the unit responsible for implementing and upholding EBP. There was no significant variation between university and non-university hospitals regarding the use of evidence-based guidelines. Only $35 \%$ of the respondents felt that management did anything in particular to promote EBP at their unit. The study suggests that evidence-based guidelines are the most commonly used vehicle for research utilisation among nurses in Danish intensive care units.

Evidence-based practice as perceived by the nurses The questionnaire contained two questions relating to the understanding of EBP. To the first question, $96 \%$ of the respondents answered that EBP is the systematic application of research-based knowledge, rather than the use of quantitative knowledge or expert knowledge. In the author's view, this was the most appropriate answer, although no conclusive definition of EBP exists. To the second question, $76 \%$ answered that EBP is limited to clinical issues, while $22 \%$ answered that EBP may include economical considerations and $2 \%$ responded that EBP does not include patient preferences. The most correct answer according to the researchers was economical considerations, but the responses reflect the lack of final definition. Table 2 shows the nurses' perceptions of EBP in daily practice. $74 \%$ of the respondents reported that they were familiar with EBP. 59\% of the respondents had consulted various literature within the past month in relation to clinical decisionmaking, and $88 \%$ perceived that EBP was relevant to nursing. $90 \%$ believed that nursing evidence exists, but only $45 \%$ felt that is was the responsibility of the individual bedside nurse to find the evidence. Finally, $96 \%$ of the respondents believed that EBP promotes the profession of nursing. The study suggests that experienced critical care nurses have a notably positive attitude towards EBP, albeit that their understanding of the phenomenon may vary.

\begin{tabular}{|c|c|c|c|}
\hline Answers given by percent & $\begin{array}{l}\text { yes } \\
\%\end{array}$ & $\begin{array}{l}\text { no } \\
\%\end{array}$ & $\begin{array}{l}\text { don't know / } \\
\text { not applicable } \\
\%\end{array}$ \\
\hline Is EBP formal policy at your hospital? & 41 & 27 & 32 \\
\hline Is EBP formal policy at your unit? & 42 & 57 & 1 \\
\hline Is an introduction course in EBP offered? & 16 & 84 & 0 \\
\hline If a course in EBP is offered, is it mandatory? & 4 & 11 & 85 \\
\hline Are evidence-based guidelines in use by the nurses? & 65 & 33 & 2 \\
\hline Is EBP the responsibility of specific nurses in your unit? & 50 & 48 & 2 \\
\hline Has management done anything in particular to promote EBP at your unit? & 35 & 63 & 2 \\
\hline
\end{tabular}

Table 1. Evidence-based practice as policy 


\begin{tabular}{|c|c|c|c|}
\hline Answers given by percent & $\begin{array}{l}\text { yes } \\
\%\end{array}$ & $\begin{array}{l}\text { no } \\
\%\end{array}$ & $\begin{array}{l}\text { don't know / } \\
\text { not applicable } \\
\%\end{array}$ \\
\hline Are you familiar with EBP? & 74 & 9 & 17 \\
\hline $\begin{array}{l}\text { Have you consulted the literature in order to make a clinical decision within the } \\
\text { past month? }\end{array}$ & 59 & 39 & 2 \\
\hline Is EBP relevant to nursing? & 88 & 5 & 7 \\
\hline Does nursing evidence exist? & 90 & 5 & 5 \\
\hline Is finding evidence the responsibility of the individual nurse? & 45 & 35 & 20 \\
\hline Does EBP promote the profession of nursing? & 96 & 2 & 2 \\
\hline
\end{tabular}

Table 2. Evidence-based practice as perceived by the nurses

\section{Sources of information}

The respondents were asked to estimate the frequency of use of various sources of information on a four-point Likert-type scale (never, rarely, regularly, almost always). The responses were ranked according to a score, which was obtained by multiplying the number of responses in each category by $1,2,3$, and 4 respectively (that is, $1 \times$ [never], $2 \times$ [rarely], $3 \times$ [regularly], and $4 \times$ [almost always] - see Table 3). Thus, the personal experience of the nurse was the most common source of information, while non-evidencebased literature along with evidence-based journals were the least frequently used sources of information. To the open-ended question of other sources of information, the respondents suggested external courses, patient narratives, protocols, and intensive care unit certification classes.

\section{Narrative comments}

The respondents were invited to comment on the individual items in the questionnaire. These comments were analysed thematically and are summarised in Figure 1.

\section{DISCUSSION}

The mean age of the participants in the study was 45 years, which is higher than the mean age of bedside intensive care unit nurses in Denmark in general, which is around 37 years (Egerod 2002). Also, the mean length of experience was 21 years as opposed to 11 years among bedside critical care nurses (Egerod 2002). Most of the respondents were clinical nurse specialists or head nurses. Arguably, this group of nurses is more likely to have knowledge of hospital policy than bedside nurses. The nurses in the survey were not a representative sample of Danish intensive care unit nurses, but they were perhaps a more reliable source of information for the purposes of this study than a random sample.

According to the respondents in this survey, EBP was perceived as part of the formal policy at less than half of the Danish hospitals. Little was done by way of introductory courses or other initiatives to encourage EBP. Only half of the units surveyed had a designated

\begin{tabular}{|c|c|c|c|c|c|c|}
\hline \multicolumn{2}{|c|}{$\begin{array}{l}\text { Number of answers by each category of information } \\
\text { source }\end{array}$} & \multirow{2}{*}{$\begin{array}{l}\text { Never } \\
0\end{array}$} & \multirow{2}{*}{$\begin{array}{l}\text { Rarely } \\
1\end{array}$} & \multirow{2}{*}{$\begin{array}{l}\text { Regularly } \\
19\end{array}$} & \multirow{2}{*}{$\begin{array}{l}\text { Almost } \\
\text { always }\end{array}$} & \multirow{2}{*}{$\begin{array}{l}\text { Score } \\
159\end{array}$} \\
\hline 1. & Personal experience & & & & & \\
\hline 2. & Textbooks & 0 & 2 & 30 & 13 & 146 \\
\hline 3. & Colleagues & 0 & 1 & 41 & 3 & 137 \\
\hline 4. & Physicians & 0 & 2 & 41 & 2 & 135 \\
\hline 5. & Hospital in-service education & 0 & 6 & 34 & 4 & 130 \\
\hline 6. & Evidence-based nursing journals in Danish & 0 & 15 & 26 & 4 & 124 \\
\hline 7. & Nursing school & 3 & 13 & 23 & 5 & 118 \\
\hline 8. & Intuition & 3 & 11 & 24 & 5 & 117 \\
\hline 9. & Patients and patients' families & 3 & 17 & 22 & 3 & 115 \\
\hline 10. & Habit or tradition & 0 & 15 & 24 & 3 & 114 \\
\hline 11. & Evidence-based nursing journals in English & 3 & 22 & 17 & 3 & 110 \\
\hline 12. & Evidence-based medical journals in Danish & 2 & 22 & 19 & 1 & 107 \\
\hline 13. & Evidence-based medical journals in English & 6 & 23 & 13 & 2 & 99 \\
\hline 14. & Non-evidence-based literature & 3 & 29 & 11 & 0 & 94 \\
\hline
\end{tabular}

(The score was obtained in each category by multiplying $1 \times$ [never], $2 \times$ [rarely], $3 \times$ [regularly], and $4 \times$ [almost always]. The scores are ranked from most to least frequent source of information.)

Table 3. Sources of information ranked from most to least frequent

* Barriers towards implementation of EBP include:

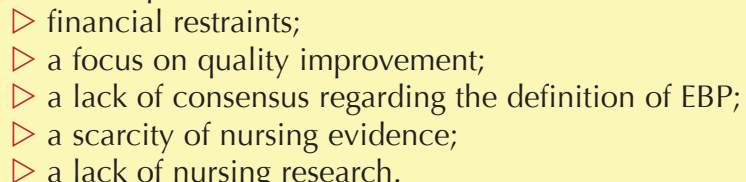

$\triangleright$ a lack of nursing research.

* $\quad$ EBP is not possible in all areas of nursing.

* The responsibility for EBP lies with management, not at the level of the individual nurse.

Figure 1. Thematic analysis of narrative comments 
nurse responsible for implementation of EBP. Most of the respondents, however, were positive about EBP while they did not regard it as the responsibility of the bedside nurse to find the evidence. Although the nurses in the survey may equate EBP with research utilisation, it appears that the most effective dissemination vehicle is the application of protocols or evidence-based guidelines. The study supports the hypothesis that Danish intensive care unit nurses rely on personal experience, or unit-based protocols where available, rather than research-based literature in relation to clinical decision-making.

The findings in this survey, regarding the sources of information used by the critical care nurses, are similar to those of a 1996 study in which Canadian bedside nurses ranked the source of their knowledge. Experiential sources, nursing school, workplace sources, colleagues, physicians, intuition, tradition, textbooks, nursing trade journals, habit, medical research journals, nursing research journals, and media sources were identified (Estabrooks 1998). It has been suggested that many nurses lack sufficient knowledge and understanding of research findings to apply research at the bedside (Hunt 1996). The respondents in the present study were highly experienced but rarely read research-based literature, especially not in English. They reported reading nursing research literature in Danish, but this type of literature is almost non-existent. They were, perhaps, alluding to the Danish nursing trade magazine Sygeplejersken (The Nurse), which does publish some research-based articles. As the respondents were mainly diploma-educated nurses, the study suggests that Danish nurses have an educational barrier as well as a language barrier when it comes to finding and appraising the evidence.

The study has shown that nurses fail to perceive information gathered from patients and their families as a primary source of knowledge, as it was ranked ninth out of 14 sources. This is noteworthy, because the intention is that nursing is aimed at the individual patient's needs. One explanation for this finding could be that nurses are not aware that the preferences of the patients play an important role in EBP (Estabrooks 1998). If EBP is equated with research utilisation, nurses may assume that only knowledge derived from bona fide scientific journals counts. A Danish study of practical learning among student nurses has demonstrated that information gathered from the patients is often unappreciated as a means of gaining knowledge (Larsen 2000). A study regarding doctors' information sources, however, shows that information is derived from the patient, the local population and medical knowledge (Smith 1996). According to this study, sources of information include the patient, the patient's family, the referring doctor, the health team and the patient record. In fact, it is stated that the art of medicine lies in gathering information of this kind. This could apply to nursing as well.

Among the narrative comments in the present study, it was stated that barriers to the implementation of EBP include financial restraints and a focus on quality improvement. This runs counter to the view that EBP has in fact been introduced to ensure quality and cost effectiveness (Bradshaw 2002). Again, this may be a sign that the nurses are unfamiliar with the motivations and interests behind the introduction of EBP, which have been described as information management, cost constraint and optimising patient care (Wharton 1999), or as a rationing mechanism (Bradshaw 2002). Some nurses in the survey pointed to the lack of nursing research as an impediment to the introduction of EBP. This view is supported by the literature (Hunt 1996), as well as the lack of consensus regarding the definition and conceptualisation of EBP (French 2002; Wang 2004). In the narrative comments, it was stated that EBP is not relevant to all areas of nursing. This is a common concern, which should not preclude the use of EBP where it is appropriate. A more important issue is that of integrating qualitative research with the overwhelmingly quantitative paradigm of EBP (DiCenso et al. 2000).

Surveys have a number of inherent limitations, such as the reliance on the respondents' estimates and recall. The population surveyed was not a random sample of Danish intensive care unit nurses. The study provided an overview of experienced nurses' perceptions of EBP, which means that the responses may not be representative of this group in general. The strength of this survey is the even representation across the country and a relatively high response rate. The survey was a pilot study for a study of cardiac nurses' use of EBP. In the next study, the nurses will be stratified so the responses of the nurse managers and bedside nurses may be compared.

\section{CONCLUSION}

This study has demonstrated that there are still many barriers to implementing EBP in Danish intensive care units, and that the problems are similar at university hospitals and non-university hospitals. Experienced critical care nurses perceive that they are familiar with EBP, but the study suggests that EBP is equated with research utilisation. Experienced critical care nurses have an overall positive attitude towards EBP but rarely rely on research-based journals as their primary source of information. Evidence-based guidelines and specially designated nurses are the most commonly used vehicles for promoting research utilisation in Danish intensive care units.

\section{REFERENCES}

Bradshaw PL. (2002) Evidence-based practice in nursing - exploring the feasibility of its implementation in Britain. Asian Journal of Nursing Studies 6 (1): 74-80.

De Vaus D. (2002) Surveys in Social Research, 5th edition. London: Routledge.

DiCenso A, Ciliska D, Marks S, McKibbon A, Cullum N \& Thompson C. (2000) Evidence-Based Nursing, In: Sackett DL, Strauss S, Richardson S, Rosenberg W \& Haynes RB (eds.) Evidence-Based Medicine. How to Practice and Teach EBM, 2nd edition. Edinburgh: Churchill Livingstone. Egerod I. (2002) Uncertain terms of sedation in ICU. Journal of Clinical Nursing 11 (6): 831-840.

Estabrooks C.A. (1998) Will evidence-based nursing practice make practice perfect? Canadian Journal of Nursing Research 30 (1): 15-36.

French P. (2002) What is the evidence on evidence-based nursing? An epistemological concern. Journal of Advanced Nursing 37 (3): 250-257.

Guyatt G \& Rennie D. (2002) Users' Guide to the Medical Literature. Chicago: American Medical Association Press.

Hunt JM. (1996) Barriers to research utilization. Journal of Advanced Nursing 23 (3): 423-425.

Larsen K. (2000) Prakitkuddannelse - kendte og miskendte sider. [Practical training - appreciated and unappreciated aspects]. Copenhagen: University Hospitals Centre for Nursing and Care Research, UCSF. PhD thesis.

Saarni SI \& Gylling HA. (2004) Evidence-based medicine guidelines: a solution to rationing or politics disguised as science? Journal of Medical 
Ethics 30 (2): 171-175.

Sackett DL, Straus SE, Richardson WS, Rosenberg W \& Haynes RB. (2000). Evidence-Based Medicine. How to Practice and Teach EBM, 2nd edition. Edinburgh: Churchill Livingstone.

Smith R. (1996) What clinical information do doctors need? British
Medical Journal 313 (7064): 1062-1068.

Wang TJ. (2004) Concept analysis of functional status. International Journal of Nursing Studies 41 (4): 457-462.

Wharton S. (1999) Defining Evidence-based medicine. University of Toronto Medical Journal 76 (3): 142-143. 